\title{
Conserving wild fish in a sea of market-based efforts
}

\author{
Jennifer Jacquet, John Hocevar, Sherman Lai, Patricia Majluf, Nathan \\ Pelletier, Tony Pitcher, Enric Sala, Rashid Sumaila and Daniel Pauly
}

\begin{abstract}
Over the past decade conservation groups have put considerable effort into educating consumers and changing patterns of household consumption. Many groups aiming to reduce overfishing and encourage sustainable fishing practices have turned to new market-based tools, including consumer awareness campaigns and seafood certification schemes (e.g. the Marine Stewardship Council) that have been well received by the fishing and fish marketing industries and by the public in many western countries. Here, we review difficulties that may impede further progress, such as consumer confusion, lack of traceability and a lack of demonstrably improved conservation status for the fish that are meant to be protected. Despite these issues, marketbased initiatives may have a place in fisheries conservation in raising awareness among consumers and in encouraging suppliers to adopt better practices. We also present several additional avenues for market-based conservation measures that may strengthen or complement current initiatives, such as working higher in the demand chain, connecting seafood security to climate change via life cycle analysis, diverting small fish away from the fishmeal industry into human food markets, and the elimination of fisheries subsidies. Finally, as was done with greenhouse gas emissions, scientists, conservation groups and governments should set seafood consumption targets.
\end{abstract}

Keywords Consumption targets, effectiveness, fisheries subsidies, fishmeal, life cycle analysis, Marine Stewardship Council, sustainable seafood, traceability

Jennifer JACQuet (Corresponding author), Sherman LAi and DANiEL Pauly Sea Around Us Project, University of British Columbia Fisheries Centre, 2202 Main Mall, Vancouver, British Columbia, Canada V6T 1Z4. E-mail j.jacquet@fisheries.ubc.ca

JOHN Hocevar Greenpeace USA, Washington, DC, USA.

Patricia Majluf Center for Environmental Sustainability, Cayetano Heredia University, Lima, Peru.

Nathan Pelletier School for Resource and Environmental Studies, Dalhousie University, Halifax, Canada.

Tony Pitcher Fisheries Ecosystems Restoration Research, University of British Columbia Fisheries Centre, Vancouver, Canada.

ENRIC SALA Center for Advanced Studies of Blanes, Blanes, Spain and National Geographic Society, Washington, DC, USA.

Rashid Sumaila University of British Columbia Fisheries Centre, Vancouver, Canada.

Received 9 March 2009. Revision requested 15 May 2009.

Accepted 18 June 2009.

\section{Introduction}

Tish consumption is on the rise globally. The USA now - consumes almost five times more fish than it did 100 years ago (c. 2.2 million $t$ in 2004 vs c. 500,000 $t$ in 1910; NMFS, 2006), and Chinese consumers are now consuming almost five times more seafood per capita than they did in 1961 (25.4 kg person ${ }^{-1}$ in 2005 vs $4.8 \mathrm{~kg}$ person $^{-1}$ in 1961; Halweil, 2006). Worldwide, per capita consumption of marine fishes has nearly doubled since the 1960 s $(9 \mathrm{~kg}$ in the 1960 s vs $16 \mathrm{~kg}$ in 1997; WHO, 2006) while the human population also doubled over this same time period. Future projections show that seafood supply from capture fisheries is decreasing and that, overall, today's marine fisheries are unsustainable (Pauly et al., 2002; Worm et al., 2006).

To meet predicted demands for seafood by 2020 aquaculture production will need to double. Alternatively, how and for what we fish (e.g. low trophic-level species for which there exists a far greater amount of biomass available for consumption) will require reform (Pitcher, 2008). Because the growing demand for seafood has been implicated in the marine fisheries crisis, it is appropriate that some effort be directed at reforming the human appetite (Grescoe, 2008). So far, this reform has largely manifested itself in a host of market efforts to encourage what is popularly referred to as 'sustainable seafood', the meaning of which is generally broad, covering ecologically responsible fishing that minimizes the bycatch of non-target species and brings acceptable levels of ecosystem and environmental impacts. Here we aim to provide an overview of these initiatives, expand upon Jacquet \& Pauly (2007) in reviewing common difficulties, and present several possible avenues for improving market-based approaches to conserve wild fish.

\section{Overview of market-based sustainable seafood initiatives for marine capture fisheries}

Marine capture fisheries peaked in the late 1980s (Watson \& Pauly, 2001), although the problem was not realized until the early 1990s. The first major seafood campaign was the boycott of canned tuna in the late 1980 s, which led to the first seafood eco-label, the 'dolphin-safe' label, in 1990 (Ward, 2008).

In 1997 the first certification scheme created specifically for sustainable fisheries was established in the form of the Marine Stewardship Council (MSC; Kaiser \& EdwardsJones, 2006). The MSC designed a set of ecological criteria for sustainable and well-managed fisheries along with a label for fish products that receive MSC approval; two 
of us (TP and DP) participated in this process. Today, the MSC label is the best established and most widely discussed fisheries certification and, at the end of 2007, c. $7 \%$ (c. 5.25 million $t$ ) of the annual global landings of marine fisheries were MSC certified.

One year after the MSC's inception the National Audubon Society and Carl Safina developed the first consumer seafood guide and, in 2000, the Monterey Bay Aquarium published a seafood wallet card. The first trade association, the Seafood Choices Alliance, founded in 2001, provided infrastructure for the initiatives that would follow (Table 1). Globally, market-based sustainable seafood initiatives are on the rise (Table 1). The fundamental goals of these initiatives are to raise awareness and ultimately reduce overexploitation of sensitive species by reducing demand (by shifting purchasing decisions towards products from more sustainable fisheries). They can be considered in the following categories (as in Roheim \& Sutinen, 2006): (1) evaluation system, i.e. criteria to determine sustainability and to generate items in (2)-(5); (2) boycotts; (3) eco-labels; (4) consumer seafood guides (including cookbooks); (5) distributor seafood guides (often used jointly with pressure on, and engagement with, retailers not to carry particular species or to improve practices); and (6) trade associations.

\section{Common difficulties}

One major concern in sustainable seafood initiatives is defining sustainability. Each initiative has a different definition of sustainability and a different methodology to assess it. A recent assessment used 18 criteria to assess nine systems for evaluating fisheries sustainability and found that there were several areas that none of them addressed adequately: (1) socio-economic attributes, (2) food security, (3) capacity of the assessment to adapt to local issues and unique aspects of the fishery, and (4) peer review of assessment decision (Leadbitter \& Ward, 2007).

Also lacking in most sustainable seafood initiatives is consideration of environmental implications of bringing both fisheries and aquaculture products to market (Pelletier \& Tyedmers, 2008). These include use efficiencies and emissions intensities of compounds such as greenhouse gases, acid precipitants, ozone-depleting and eutrophication agents and a wide variety of chemicals with ecotoxicological effects (Pelletier et al., 2006). A growing body of life cycle and energy analyses of fisheries and aquaculture identify the key stages of seafood supply chains and facilitate comparisons of eco-efficiency between competing production technologies (Pelletier \& Tyedmers, 2008).

The difficulties associated with assessing and communicating sustainability also manifest themselves in consumer confusion. For instance, the 2007 Seafood Watch wallet card from Monterey Bay Aquarium lists 12 different tuna types (i.e. species, method of fishing, country) between the three columns of 'best choices', 'good alternatives' and 'avoid'. WWF's seafood choice card in Spain lists bluefin tuna (Thunnus thynnus, Thunnus maccoyii or Thunnus orientalis) as species to avoid but as a 'best choice' if the tuna come from coastal fishing traps. Although these complexities reflect the reality of the global seafood market, they also confuse consumers (Jacquet, 2007). This confusion is intensified by government agencies recommending the consumption of seafood because of its nutritional value (Brunner et al., 2009) at the same time as other agencies recommend eating less of certain species because of their high concentrations of mercury and other toxic compounds (e.g. Environmental Defense Fund's Seafood Selector; Environmental Defense Fund, 2009). In addition, many seafood guides have a regional focus, despite the global nature of the seafood market. In the USA consumer seafood guides focus mainly on fish caught by national fisheries (Roheim \& Sutinen, 2006), which is inappropriate, given that the USA imports $>80 \%$ of its seafood.

Furthermore, different consumer guides provide different recommendations depending on their criteria for ranking seafood, so that there is confusion generated not only by each card but also between cards. Each sustainable seafood initiative must have some way of assessing sustainability, which invariably leads to inconsistencies. This confusion is evident at the International Seafood Guide (2009), which was intended to enable the querying of seafood guides by web-enabled phones (R. Froese, IFM-GEOMAR, Kiel, Germany, pers. comm.) but has inadvertently exposed inconsistencies between the guides it covers. The website presents seafood guides for 19 countries and displays interesting results for countries with more than one guide (e.g. the website presents three for Germany and nine for the USA). While different organizations may generate a unanimous recommendation (i.e. 'avoid') for some species (e.g. Atlantic cod Gadus morhua, Atlantic bluefin tuna T. thynnus), the recommendations can be inconsistent for other species. For example, six organizations (including the Blue Ocean Institute), rank Atlantic halibut Hippoglossus hippoglossus as a fish to avoid, whereas Environmental Defense recommends consuming it with caution and Friends of the Sea and Monterey Bay recommend it as a sustainable choice. Similarly, inconsistent recommendations are generated for albacore Thunnus alalunga, bigeye tuna Thunnus obesus, lingcod Ophiodon elongatus, Atlantic haddock Melanogrammus aeglefinus and many other species.

Consumer boycotts and consumer guides have also been criticized for being indiscriminate towards responsible and irresponsible fishing operators, imposing an economic cost on responsible operators (Kaiser \& Edwards-Jones, 2006; Roheim \& Sutinen, 2006). It has been argued that ecolabelling, on the other hand, is capable of rewarding individual operators because it engages consumers in rewarding certain members or sub-sectors of the fishing community 
TABLE 1 Timeline and overview of market-based sustainable seafood initiatives (all internet addresses were correct at 15 February 2009).

\begin{tabular}{|c|c|c|c|c|c|}
\hline Year & Country & Sponsor & Category & Name & Remarks/URL \\
\hline $\begin{array}{l}\text { Late } \\
1980 \mathrm{~s}\end{array}$ & USA & $\begin{array}{l}\text { Earth Island } \\
\text { Institute }\end{array}$ & Boycott/eco-label & Dolphin-safe logo & $\begin{array}{l}\text { Boycott of canned tuna resulted in the } \\
\text { first seafood eco-label, the 'dolphin-safe' } \\
\text { logo; http://www.earthisland.org/ } \\
\text { dolphinSafeTuna/consumer/ }\end{array}$ \\
\hline 1995 & Global & $\begin{array}{l}\text { UN Food \& } \\
\text { Agriculture } \\
\text { Organization } \\
\text { (FAO) }\end{array}$ & Evaluation system & $\begin{array}{l}\text { Code of Conduct for } \\
\text { Responsible Fisheries }\end{array}$ & $\begin{array}{l}\text { Voluntary agreement that sets out } \\
\text { principles \& international standards for } \\
\text { fisheries management; http://www.fao. } \\
\text { org/DOCREP/005/v9878e/v9878e00.htm }\end{array}$ \\
\hline 1997 & Global & $\begin{array}{l}\text { WWF \& } \\
\text { Unilever }\end{array}$ & $\begin{array}{l}\text { Eco-label/evaluation } \\
\text { system }\end{array}$ & $\begin{array}{l}\text { Marine Stewardship } \\
\text { Council (MSC) }\end{array}$ & $\begin{array}{l}\text { Became independent in 1999; } \\
\text { http://www.msc.org }\end{array}$ \\
\hline 1997 & USA & $\begin{array}{l}\text { Monterey Bay } \\
\text { Aquarium }\end{array}$ & $\begin{array}{l}\text { Consumer seafood } \\
\text { guide/evaluation } \\
\text { system }\end{array}$ & $\begin{array}{l}\text { Seafood Watch } \\
\text { Program }\end{array}$ & $\begin{array}{l}\text { Began as a list of sustainable seafood } \\
\text { for an aquarium exhibit \& in } 2000 \\
\text { became a wallet card; other aquaria } \\
\text { use the same information with a } \\
\text { different interface; http://www.mbayaq. } \\
\text { org/cr/seafoodwatch.asp }\end{array}$ \\
\hline 1998 & USA & $\begin{array}{l}\text { National } \\
\text { Audubon } \\
\text { Society }\end{array}$ & $\begin{array}{l}\text { Consumer seafood } \\
\text { guide/evaluation } \\
\text { system }\end{array}$ & $\begin{array}{l}\text { Seafood Lover's } \\
\text { Guide }\end{array}$ & $\begin{array}{l}\text { First consumer seafood guide, produced } \\
\text { by Carl Safina who, at the time, was } \\
\text { working for Audubon; today, it is } \\
\text { also co-sponsored by the Wildlife } \\
\text { Conservation Society; http://seafood. } \\
\text { audubon.org/ }\end{array}$ \\
\hline $\begin{array}{l}1998- \\
2000\end{array}$ & USA & $\begin{array}{l}\text { SeaWeb \& } \\
\text { Natural } \\
\text { Resources } \\
\text { Defense } \\
\text { Council }\end{array}$ & Boycott & $\begin{array}{l}\text { Give Swordfish a } \\
\text { Break }\end{array}$ & $\begin{array}{l}700 \text { chefs boycott swordfish until the } \\
\text { international fishery commission cut } \\
\text { quotas; http://www.seaweb.org/ } \\
\text { programs/swordfish/ }\end{array}$ \\
\hline 1999 & Global & & Evaluation system & RapFish & $\begin{array}{l}\text { RapFish considers five areas: ecological, } \\
\text { economic, ethical, social \& technological } \\
\text { (Pitcher \& Preikshot, 2001) as well as } \\
\text { the FAO Code of Conduct (Pitcher, } \\
\text { 1999); Microsoft Excel software for } \\
\text { RapFish is available (Kavanagh \& } \\
\text { Pitcher, 2004); http://www.fao.org/ } \\
\text { DOCREP/005/X4175E/X4175E00.HTM }\end{array}$ \\
\hline 1999 & USA & & $\begin{array}{l}\text { Eco-label/evaluation } \\
\text { system }\end{array}$ & EcoFish & $\begin{array}{l}\text { New England-based company selling } \\
\text { sustainably caught fish; in } 2001 \text { EcoFish } \\
\text { was certified as sustainable by the MSC } \\
\text { (the first seafood distributor to earn } \\
\text { such certification); also have a new } \\
\text { seafood-safe logo based on contaminant } \\
\text { testing; http://www.ecofish.com/ }\end{array}$ \\
\hline 1999 & Australia & & Evaluation system & $\begin{array}{l}\text { Environment } \\
\text { Protection } \\
\text { \& Biodiversity } \\
\text { Conservation Act }\end{array}$ & $\begin{array}{l}\text { Legislation that requires fisheries that } \\
\text { fall into certain categories to undergo } \\
\text { sustainability assessments that are } \\
\text { then submitted to the Department of } \\
\text { Environment \& Heritage (has been } \\
\text { done for nearly every export species); } \\
\text { http://www.environment.gov.au/epbc/ }\end{array}$ \\
\hline 1999 & Australia & & $\begin{array}{l}\text { Consumer seafood } \\
\text { guide/evaluation } \\
\text { system }\end{array}$ & $\begin{array}{l}\text { Victoria National } \\
\text { Parks Association }\end{array}$ & $\begin{array}{l}\text { Evaluates the status of important } \\
\text { commercial fishery species from } \\
\text { southern Australia }\end{array}$ \\
\hline 2000 & USA & & $\begin{array}{l}\text { Consumer seafood } \\
\text { guide }\end{array}$ & $\begin{array}{l}\text { Fish for Thought: } \\
\text { An Eco-Cookbook }\end{array}$ & \\
\hline 2001 & USA & & Trade association & $\begin{array}{l}\text { Seafood Choices } \\
\text { Alliance }\end{array}$ & $\begin{array}{l}\text { Http://www.seafoodchoices. } \\
\text { com/home.php }\end{array}$ \\
\hline
\end{tabular}


TABLE 1 (Continued)

\begin{tabular}{|c|c|c|c|c|c|}
\hline Year & Country & Sponsor & Category & Name & Remarks/URL \\
\hline 2001 & USA & $\begin{array}{l}\text { Environmental } \\
\text { Defense }\end{array}$ & $\begin{array}{l}\text { Consumer seafood } \\
\text { guide }\end{array}$ & Oceans Alive & $\begin{array}{l}\text { Programme was formerly known as } \\
\text { Seafood Selector Website \& Pocket } \\
\text { Guide \& was renamed the Oceans } \\
\text { Alive programme in 2005; http://www. } \\
\text { oceansalive.org/home.cfm }\end{array}$ \\
\hline 2001 & USA & EarthEasy & $\begin{array}{l}\text { Consumer seafood } \\
\text { guide }\end{array}$ & $\begin{array}{l}\text { Sustainable Seafood } \\
\text { Guide }\end{array}$ & $\begin{array}{l}\text { Http://www.eartheasy.com/eat_ } \\
\text { sustainable_seafoods.htm }\end{array}$ \\
\hline 2001 & USA & $\begin{array}{l}\text { Seafood Choices } \\
\text { Alliance }\end{array}$ & $\begin{array}{l}\text { Distributor seafood } \\
\text { guide }\end{array}$ & Sourcing Seafood & $\begin{array}{l}\text { Http://www.seafoodchoices.com/ } \\
\text { resources/sourcingseafood.php }\end{array}$ \\
\hline 2001 & USA & & $\begin{array}{l}\text { Distributor seafood } \\
\text { guide }\end{array}$ & Choice Catch & $\begin{array}{l}\text { Sponsored by New England Aquarium } \\
\text { \& Ahold; formerly known as EcoSounds, } \\
\text { Choice Catch audits domestic \& } \\
\text { international sources of seafood for } \\
\text { environmental impact \& sustainability } \\
\text { based on scientific information } \\
\text { available to ensure sustainability } \\
\text { \& traceability of Ahold's seafood } \\
\text { products (Ahold is the fifth largest US } \\
\text { grocery chain); http://www.neaq.org/ } \\
\text { choicecatch/ }\end{array}$ \\
\hline 2001 & Global & & $\begin{array}{l}\text { Eco-label/evaluation } \\
\text { system }\end{array}$ & $\begin{array}{l}\text { Marine Aquarium } \\
\text { Council certification } \\
\text { \& eco-label }\end{array}$ & $\begin{array}{l}\text { Marine Aquarium Council is a } \\
\text { third-party certifier; http://www. } \\
\text { aquariumcouncil.org/ }\end{array}$ \\
\hline 2002 & Global & & Evaluation system & Traffic Lights & $\begin{array}{l}\text { Unilever's internal fishery assessment } \\
\text { system based on FAO guidelines; } \\
\text { http://www.seafoodchoices.com/ } \\
\text { membership/champions_unilever.php }\end{array}$ \\
\hline $\begin{array}{l}2002- \\
2007\end{array}$ & USA & $\begin{array}{l}\text { National } \\
\text { Environmental } \\
\text { Trust (NET) }\end{array}$ & Boycott & $\begin{array}{l}\text { Take a Pass on } \\
\text { Chilean Sea } \\
\text { Bass campaign }\end{array}$ & $\begin{array}{l}\text { Programme ended when } \\
\text { NET merged with Pew } \\
\text { Charitable Trusts }\end{array}$ \\
\hline 2002 & USA & $\begin{array}{l}\text { Environmental } \\
\text { Defense }\end{array}$ & $\begin{array}{l}\text { Distributor seafood } \\
\text { guide }\end{array}$ & $\begin{array}{l}\text { Business Guide to } \\
\text { Sustainable Seafood }\end{array}$ & $\begin{array}{l}\text { 101-page document available at http:// } \\
\text { www.environmentaldefense.org/ } \\
\text { documents/2532_BusinessGuide } \\
\text { SustainableSeafood.pdf }\end{array}$ \\
\hline 2002 & USA & $\begin{array}{l}\text { Sustainable } \\
\text { Fishery } \\
\text { Advocates }\end{array}$ & Eco-label & FishWise & $\begin{array}{l}\text { Began in California \& is now } \\
\text { implemented in } 34 \text { stores; as of } 2005 \\
\text { also provides retailers/consumers with } \\
\text { information regarding contaminants; } \\
\text { http://www.sustainablefishery.org/ }\end{array}$ \\
\hline 2002 & USA & $\begin{array}{l}\text { South Carolina } \\
\text { Aquarium }\end{array}$ & $\begin{array}{l}\text { Distributor seafood } \\
\text { guide }\end{array}$ & $\begin{array}{l}\text { Sustainable } \\
\text { Seafood Initiative }\end{array}$ & $\begin{array}{l}\text { Restaurant partners agree to remove } \\
\text { Chilean sea bass, orange roughy \& } \\
\text { shark from their menus; collaboration } \\
\text { between southern business \& NGOs; } \\
\text { http://www.scaquarium.org/SSI/ } \\
\text { default.html }\end{array}$ \\
\hline 2002 & UK & $\begin{array}{l}\text { Marine } \\
\text { Conservation } \\
\text { Society }\end{array}$ & $\begin{array}{l}\text { Distributor seafood } \\
\text { guide }\end{array}$ & Good Fish Guide & $\begin{array}{l}\text { Has been used by some UK } \\
\text { supermarkets looking at sustainability; } \\
\text { http://www.fishonline.org/ }\end{array}$ \\
\hline $\begin{array}{r}2002- \\
2005\end{array}$ & Canada & $\begin{array}{l}\text { Endangered Fish } \\
\text { Alliance }\end{array}$ & $\begin{array}{l}\text { Distributor seafood } \\
\text { guide }\end{array}$ & $\begin{array}{l}\text { Endangered } \\
\text { Fish Alliance }\end{array}$ & $\begin{array}{l}\text { Ask that members not serve swordfish, } \\
\text { Chilean sea bass, orange roughy \& } \\
\text { certain types of caviar; } 161 \text { pioneer } \\
\text { members; in } 2005 \text { merged with } \\
\text { Environmental Defense; http://www. } \\
\text { endangeredfishalliance.org/ }\end{array}$ \\
\hline
\end{tabular}


TABLE 1 (Continued)

\begin{tabular}{|c|c|c|c|c|c|}
\hline Year & Country & Sponsor & Category & Name & Remarks/URL \\
\hline 2003 & USA & $\begin{array}{l}\text { Blue Ocean } \\
\text { Institute }\end{array}$ & $\begin{array}{l}\text { Consumer seafood } \\
\text { guide/evaluation } \\
\text { system }\end{array}$ & & $\begin{array}{l}\text { Fully transparent; based on the } \\
\text { methodology developed by Carl Safina } \\
\text { in the Audubon Society's Seafood } \\
\text { Lover's Almanac; other aquariums use } \\
\text { the same information with a different } \\
\text { interface (e.g. Shedd aquarium relabels } \\
\text { it as Right Bite) }\end{array}$ \\
\hline 2004 & USA & Star Chefs & $\begin{array}{l}\text { Consumer seafood } \\
\text { guide }\end{array}$ & $\begin{array}{l}\text { Sustainable } \\
\text { Seafood Guide }\end{array}$ & $\begin{array}{l}\text { Http://www.starchefs.com/features/ } \\
\text { food_debates/html/sustainable_seafood. } \\
\text { shtml }\end{array}$ \\
\hline 2004 & Canada & & Eco-label? & Organic Ocean & $\begin{array}{l}\text { Group of independent British Columbia } \\
\text { fishermen who sell sustainable seafood; } \\
\text { Http://www.organicocean.com/ }\end{array}$ \\
\hline 2004 & New Zealand & $\begin{array}{l}\text { New Zealand } \\
\text { Forest \& Bird }\end{array}$ & $\begin{array}{l}\text { Consumer seafood } \\
\text { guide/evaluation } \\
\text { system }\end{array}$ & Best Fish Guide & $\begin{array}{l}\text { Have full evaluation online; first } \\
\text { independent ecological ranking for } \\
\text { New Zealand commercial fisheries; } \\
\text { http://www.forestandbird.org.nz/ } \\
\text { bestfishguide/index.asp }\end{array}$ \\
\hline 2004 & USA & $\begin{array}{l}\text { The Smithsonian } \\
\text { Institution }\end{array}$ & $\begin{array}{l}\text { Consumer seafood } \\
\text { guide }\end{array}$ & $\begin{array}{l}\text { One Fish, Two Fish, } \\
\text { Crawfish, Bluefish }\end{array}$ & $\begin{array}{l}\text { Sustainable seafood } \\
\text { cookbook }\end{array}$ \\
\hline 2004 & Australia & $\begin{array}{l}\text { Marine } \\
\text { Conservation } \\
\text { Society }\end{array}$ & $\begin{array}{l}\text { Consumer seafood } \\
\text { guide }\end{array}$ & $\begin{array}{l}\text { Sustainable Seafood } \\
\text { Guide }\end{array}$ & $\begin{array}{l}\text { Http://www.amcs.org.au/default2. } \\
\text { asp?active_page_id }=137\end{array}$ \\
\hline 2004 & South Africa & WWF & $\begin{array}{l}\text { Consumer seafood } \\
\text { guide }\end{array}$ & Seafood pocket guide & $\begin{array}{l}\text { Text messaging available; } \\
\text { http://www.panda.org.za/sassi/ }\end{array}$ \\
\hline 2005 & USA & & $\begin{array}{l}\text { Consumer seafood } \\
\text { guide }\end{array}$ & $\begin{array}{l}\text { Co-op American Safe } \\
\text { Seafood Wallet List }\end{array}$ & $\begin{array}{l}\text { Article that combined information } \\
\text { from the Food \& Drug Administration, } \\
\text { Monterey Bay Aquarium \& Blue Ocean } \\
\text { Institute; reprinted by other magazines }\end{array}$ \\
\hline 2005 & $\begin{array}{l}\text { European } \\
\text { Union }\end{array}$ & & Trade association & $\begin{array}{l}\text { Seafood Choices } \\
\text { Alliance }\end{array}$ & European branch \\
\hline 2005 & UK & Greenpeace & $\begin{array}{l}\text { Distributor seafood } \\
\text { guide }\end{array}$ & Recipe for Disaster & $\begin{array}{l}\text { Reviews sustainability of common } \\
\text { fish in UK supermarkets by combining } \\
\text { recommendations from Australian, } \\
\text { New Zealand, USA \& UK NGOs }\end{array}$ \\
\hline 2005 & $\begin{array}{l}\text { Italy, } \\
\text { Switzerland }\end{array}$ & $\begin{array}{l}\text { Friends of } \\
\text { the Sea }\end{array}$ & $\begin{array}{l}\text { Eco-label/evaluation } \\
\text { system }\end{array}$ & & $\begin{array}{l}\text { Uses FAO guidelines \& third-party } \\
\text { certification (includes aquaculture) \& is } \\
\text { used by Italy-based retailer Co-op \&, in } \\
\text { October } 2007 \text {, the Swiss retailer } \\
\text { MANOR; also allows text messaging }\end{array}$ \\
\hline 2005 & Canada & $\begin{array}{l}\text { Vancouver } \\
\text { Aquarium }\end{array}$ & $\begin{array}{l}\text { Distributor seafood } \\
\text { guide/evaluation } \\
\text { system/eco-label }\end{array}$ & Ocean Wise & Vancouver-based restaurants \\
\hline 2005 & Spain & Greenpeace & $\begin{array}{l}\text { Consumer seafood } \\
\text { guide }\end{array}$ & $\begin{array}{l}\text { Guia para el consumo } \\
\text { responsable } \\
\text { de pescado }\end{array}$ & Report \& consumer guide \\
\hline 2006 & USA & $\begin{array}{l}\text { PCC Natural } \\
\text { Markets }\end{array}$ & $\begin{array}{l}\text { Consumer seafood } \\
\text { guide }\end{array}$ & $\begin{array}{l}\text { Seafood choices for } \\
\text { a healthier ocean }\end{array}$ & $\begin{array}{l}\text { Partnership with Monterey } \\
\text { Bay Aquarium }\end{array}$ \\
\hline 2006 & USA & $\begin{array}{l}\text { Environmental } \\
\text { Defense, } \\
\text { Wegman's \& } \\
\text { Bon Appetit }\end{array}$ & $\begin{array}{l}\text { Distributor seafood } \\
\text { guide }\end{array}$ & $\begin{array}{l}\text { First corporate } \\
\text { purchasing } \\
\text { partnership }\end{array}$ & $\begin{array}{l}\text { Http://www.environmentaldefense.org/ } \\
\text { page.cfm?tagID }=1464\end{array}$ \\
\hline 2006 & Canada & Various NGOs & $\begin{array}{l}\text { Consumer seafood } \\
\text { guide/evaluation } \\
\text { system }\end{array}$ & SeaChoice & \\
\hline
\end{tabular}


TABLE 1 (Continued)

\begin{tabular}{|c|c|c|c|c|c|}
\hline Year & Country & Sponsor & Category & Name & Remarks/URL \\
\hline 2006 & $\begin{array}{l}\text { European } \\
\text { Union }\end{array}$ & IncoFish Project & Miscellaneous & FisherMin & $\begin{array}{l}\text { A ruler against which shoppers can } \\
\text { measure their fish to ensure they are } \\
\text { not buying juveniles (Froese, 2004); } \\
\text { http://www.incofish.org/Results/tools/ } \\
\text { FishNorth.php }\end{array}$ \\
\hline 2007 & $\begin{array}{l}\text { European } \\
\text { Union/N } \\
\text { America }\end{array}$ & $\begin{array}{l}\text { WWF, the North } \\
\text { Sea Foundation } \\
\text { (Dutch), } \\
\text { Greenpeace, } \\
\text { Seafood } \\
\text { Choices Alliance }\end{array}$ & Evaluation system & $\begin{array}{l}\text { A Common Tool for } \\
\text { Assessing Seafood } \\
\text { Sustainability }\end{array}$ & $\begin{array}{l}\text { Http://www.seafoodchoices. } \\
\text { org/newsroom/Common } \\
\text { Methods.php }\end{array}$ \\
\hline 2007 & $\begin{array}{l}\text { European } \\
\text { Union/North } \\
\text { America }\end{array}$ & & $\begin{array}{l}\text { Distributor seafood } \\
\text { guide }\end{array}$ & $\begin{array}{l}\text { Sustainable Fisheries } \\
\text { Partnership/FishSource }\end{array}$ & \\
\hline 2007 & European Union & & Trade Association & $\begin{array}{l}\text { Responsible Fishing } \\
\text { Alliance }\end{array}$ & $\begin{array}{l}\text { Sustainable Food Lab will work with } \\
\text { business-to-business seafood trade } \\
\text { focusing on small-scale capture } \\
\text { fishers \& aquaculture }\end{array}$ \\
\hline 2007 & USA & $\begin{array}{l}\text { Blue Ocean } \\
\text { Institute }\end{array}$ & Consumer seafood guide & Fish Phone & \\
\hline 2007 & Belgium & WWF & Consumer seafood guide & & \\
\hline 2007 & Denmark & WWF & Consumer seafood guide & & \\
\hline 2007 & Finland & WWF & Consumer seafood guide & & \\
\hline 2007 & France & WWF & Consumer seafood guide & & \\
\hline 2007 & Germany & WWF & Consumer seafood guide & & \\
\hline 2007 & Hong Kong & WWF & Consumer seafood guide & & \\
\hline 2007 & Indonesia & WWF & Consumer seafood guide & & \\
\hline 2007 & The Netherlands & WWF & Consumer seafood guide & & \\
\hline 2007 & Norway & WWF & Consumer seafood guide & & \\
\hline 2007 & Poland & WWF & Consumer seafood guide & & \\
\hline 2007 & Spain & WWF & Consumer seafood guide & & \\
\hline 2007 & Sweden & WWF & Consumer seafood guide & & \\
\hline 2007 & Switzerland & WWF & Consumer seafood guide & & \\
\hline$?$ & $\begin{array}{l}\text { The } \\
\text { Netherlands }\end{array}$ & $\begin{array}{l}\text { North Sea } \\
\text { Foundation }\end{array}$ & Consumer seafood guide & $\begin{array}{l}\text { North Sea Foundation } \\
\text { De Goede Visgids }\end{array}$ & Http://www.goedevis.nl/ \\
\hline 2002 & Germany & Greenpeace & Consumer seafood guide & Magazin & $\begin{array}{l}\text { Http://www.greenpeace.de/themen/ } \\
\text { meere/fischerei/ }\end{array}$ \\
\hline 2008 & $\begin{array}{l}\text { The } \\
\text { Netherlands }\end{array}$ & Greenpeace & Consumer seafood guide & Maak Schoon Schap & Http://www.maakschoonschap.nl/ \\
\hline 2008 & USA & $\begin{array}{l}\text { New England } \\
\text { Aquarium }\end{array}$ & Consumer seafood guide & Celebrate Seafood & $\begin{array}{l}\text { Http://neaq.org/documents/ } \\
\text { CelebrateSeafoodGuide.pdf }\end{array}$ \\
\hline
\end{tabular}

who conduct responsible fishing practices (Roheim \& Sutinen, 2006). Unfortunately, rewarding individual operators may also be at odds with creating simple messages. The MSC certification of the fishery for Patagonian toothfish Dissostichus eleginoides in British-controlled waters off South Georgia, for instance, rewarded responsible fishing operators in that one region but sent a mixed message to consumers who were simultaneously being advised by the US-based National Environmental Trust to boycott the fish (Jacquet, 2007).

Other points of concern for the MSC eco-label are the lack of consideration of stock declines, key life history characteristics, food chain, bycatch and habitat effects and/ or a failure to identify all stakeholders (for instance, in the MSC's certification of Alaska pollock Theragra chalcogramma, New Zealand hoki Macruronus novaezelandiae and Western Australian rock lobster Panulirus cygnus; Ward, 2008). Moreover, another round of controversy could begin if the MSC certifies fisheries that target fish to reduce to animal feed because of the wastefulness of converting wild fish into smaller, but more valuable, amounts of farmed fish.

Other debates have arisen over the MSC and other ecolabels because they cannot accommodate small-scale fisheries (Jacquet \& Pauly, 2008a; Gulbrandsen, 2009). Small-scale 
fisheries are probably the best hope for sustainable fisheries as they typically favour passive gears (which catch fewer unwanted species), although there are exceptions to this. On a global scale, small-scale fisheries employ 25 times more people and use $25 \%$ of the fuel to catch approximately the same amount of fish for human consumption as the large-scale industrial fishing sector (Chuenpagdee et al., 2006). But in the marketplace small-scale fisheries are at a disadvantage when competing for market access with large-scale industrial fleets, which are often heavily subsidized (Sumaila \& Pauly, 2006).

For seafood that is not MSC certified, which is $>90 \%$ of seafood consumed globally, traceability is also an issue. For seafood awareness programmes to obtain their desired outcomes it is essential that seafood consumers have accurate information and that eco-labels acquire and/or maintain their integrity. But in the USA more than one third of all fish appear to be mislabelled (Tennyson et al., 1997) and many fish are also renamed. Mislabelling and renaming subvert sustainable seafood initiatives, including those aimed at consumers (Jacquet \& Pauly, 2008b).

After distributing $>_{1}$ million seafood wallet cards, the Monterey Bay Aquarium conducted a study that revealed no overall change in the market and that fishing pressures had not decreased for targeted species (Quadra Planning Consultants Ltd, 2004). A review of 30 non-profit organizations with market-based sustainable seafood campaigns during 1999-2004 found that consumer-oriented campaigns increased awareness and visibility for the sustainable seafood issue but that there is 'no clear evidence that this increased salience is leading to big changes in buying practices, nor accelerated fisheries policies' (Bridgespan Group, 2005).

Eco-labels may also not have the impact conservationists desire. The dolphin-safe tuna label is often perceived as the most effective seafood-related label. The label, combined with several national and international regulatory schemes (e.g. the US Marine Mammal Protection Act, the US embargo on non-dolphin-safe tuna and the International Dolphin Conservation Act), has helped reduce dolphin mortality because of a reduction in the number of boats setting on dolphins, and certain populations, such as the bottlenose Tursiops truncates and short-beaked common dolphins Delphinus delphis in the eastern tropical Pacific, have increased (Gerrodette et al., 2008). This is why, in 2003, NOAA fisheries scientists suggested the definition of the label not be watered down, under international political pressure, to allow tuna caught with chasing-and-encircling techniques to be certified as 'dolphin safe'.

However, several dolphin populations have not recovered as quickly as expected. For instance, time series data of population estimates for the north-eastern pan-tropical spotted dolphin Stenella attenuata attenuata and eastern spinner dolphin Stenella longirostris populations in the eastern tropical Pacific from 1978 to 2000 show essentially no change in abundance over that time (Gerrodette \& Forcada, 2005). There does appear to have been a slight increase in the abundance of the two species in recent years (Gerrodette et al., 2008) but spotted dolphins and spinner dolphins are estimated to be at 19 and $29 \%$, respectively, of their abundance levels prior to 1959, the year when a yellowfin tuna Thunnus albacares purse seine fishery began in their region (Wade et al., 2007). The tuna purse seine fishery continues to have negative effects on dolphin populations because of unreported bycatch, the separation of mothers and calves and/or other factors (Gerrodette \& Forcada, 2005; Cramer et al., 2008). These data, along with Ward (2008), show that certain dolphin populations are still in trouble, despite the label, $100 \%$ observer coverage and strict regulations, because so-called dolphin-safe methods of catching tuna did not lead to a sufficient reduction in overall fishing pressure and hence bycatch.

Furthermore, MSC certification is unlikely to arrest the decline in fish stocks (Ward, 2008; Gulbrandsen, 2009). There are many concerns over the MSC-certified Alaska pollock fishery, which has been regarded as a poster fishery for sustainable seafood. For example, pollock recruitment data are below average in 6 of the past 7 years, fishers are travelling greater distances to find fish and northern fur seals Callorhinus ursinus, which feed on pollock, are in decline. Acoustic surveys indicated that the 2008 pollock biomass was almost $50 \%$ below the biomass from the previous year, prompting NOAA officials to recommend to the North Pacific Fishery Management Council a sustainable catch of 815,000 metric $t$ for 2009 , an $18.5 \%$ reduction from 2008 (NOAA, 2008a). In some cases, such as in Western Australia, MSC certification may even be giving fishing interests leverage against establishing fishing sanctuaries on the grounds that sanctuaries are unnecessary if the affected fisheries are already certified (Sutton, 2003).

In the USA, the country where most consumer-based efforts focus, per capita demand for seafood has increased from $6.9 \mathrm{~kg}$ person ${ }^{-1}$ in 2000 to $7.4 \mathrm{~kg} \mathrm{person}^{-1}$ in 2007 (NOAA, 2008b). Many of the species considered least sustainable by conservation groups (e.g. shrimp, tuna and salmon) remain the most desirable (perhaps because consumers are more attracted to rare species than common ones; Gault et al., 2008; Angulo \& Courchamp, 2009). Whereas there may be regional exceptions, overall, eco-labels on behalf of dolphins and fisheries appear to be weak instruments for conservation (Ward, 2008) and consumer campaigns do not seem effective at reducing demand or fishing pressure.

\section{More effective market-based efforts to conserve wild fish?}

Given the limitations of consumer-based efforts to promote sustainable seafood, perhaps the large investment in the household consumer sector was premature. Based on our 
collective experience and evidence, we present several additional avenues for market-based conservation measures that may strengthen or complement current initiatives. We also argue that these proposed ideas should be tested in accordance with scientific methods and evidence-based conservation (Sutherland et al., 2004).

\section{Labelling standards}

Consumers cannot always be sure that the seafood they purchase is the fish that the label or menu claims (Jacquet \& Pauly, 2008b). Without access to information on country of origin (where the fish is caught, not processed) and fishing method, let alone the correct species, consumers cannot make effective decisions on behalf of sustainable seafood. Therefore, all market-based initiatives must consider putting additional effort into improving labelling standards.

Of all the sustainable seafood initiatives the MSC offers the best model of traceability in the form of chain of custody standards, which traces fish from the fishery of origin to the point of sale. There is evidence that traceability standards in fisheries (e.g. South Georgia Patagonian toothfish) have improved as a result of MSC certification (Roheim \& Sutinen, 2006). Without proper labelling, consumer-oriented campaigns will continue to be undermined by cheating.

\section{Directing efforts higher than households}

In recognition of the limitations and pace of affecting household consumers, efforts to promote sustainable seafood should aim higher in the demand chain and focus on affecting large buyers. Focusing on large retailers is the new goal of Seafood Choices (Bridgespan Group, 2005) and several other groups (Table 1). In May 2008, for instance, 14 American and Canadian organizations formed the Conservation Alliance for Seafood Solutions and released their Common Vision for Environmentally Sustainable Seafood. Among food retailers the 10 largest companies account for nearly $50 \%$ of the US seafood market (Bridgespan Group, 2005). In the UK nearly $90 \%$ of seafood is now sold through supermarkets, which have gained immense buying power, particularly compared to formerly, when fish was primarily sold through local fishmongers (Greenpeace, 2005). This focus on targeting large retailers, which consolidate seafood purchases, is likely to have a bigger and faster market impact than consumer guides. Furthermore, large retailers appear concerned about wild fish stocks on the grounds that sustainability is good business and perhaps also for the sake of an increase in reputation.

\section{Balancing positive and negative messaging in the marketplace}

While it is true that environmental NGOs can use retailers as allies to force change, another strategy that is underused, particularly in North America, is the use of negative messaging to affect retailer reputation. According to research related to cooperation, a good reputation is valuable currency and is gained by playing by the rules of a social community. On the other hand, uncooperative behaviour may be profitable unless it negatively affects reputation (Semmann et al., 2004). Also, whenever individual or institutional behaviour is relevant to the public good, it should be made public (Pfeiffer \& Nowak, 2006). Under these premises, one way to motivate large seafood retailers is to generate bad press that highlights unsustainable practices. This negative messaging uses the base of consumer awareness that has been raised by wallet cards and eco-labels to push companies already engaged in sustainability efforts to step up their efforts.

Greenpeace is one group using reputation in marketbased seafood efforts. Firstly in Europe (in the UK, followed by the Netherlands, Denmark, Germany, France, Spain, Austria and Norway) and most recently in the USA, Greenpeace has used a ranking system to assess supermarket chains in terms of the sustainability of their seafood (Greenpeace, 2005, 2006). The ranking has created competition at all levels, with some retailers taking steps to try to get to the top of the ranking and others seeking to get off the bottom. In the lead-up to the publication of Greenpeace's ranking of US retailers, top-scoring Whole Foods Market agreed to stop selling red-listed orange roughy Hoplosthesus atlanticus, Target committed to dropping red snapper Lutjanus campechanus and Wegman's dropped bluefin tuna. In every country where Greenpeace ranked retailers, several retailers adopted sustainable seafood procurement policies and dropped several red-listed items. Within just 2 months of the release of the Swedish report, all but one major Swedish retailer had dropped all 14 products on Greenpeace Nordic's Red List.

Greenpeace also uses public displays to affect reputation and catalyse change in seafood markets. In November 2008 Greenpeace erected 'crime scenes' at eight Loblaw grocery stores in Toronto with the message 'caught red-handed selling Red-List fish'. The following day Loblaw officials were reportedly 'disappointed' but said they would work to offer more MSC-certified fish (Fiorillo, 2008). The more conventional methods of positive messaging and alliance with retailers are complemented (and could be even more so) using negative messaging and reputation to motivate large seafood retailers to act.

\section{Connecting seafood to climate change}

The concern for global warming presents opportunities to connect seafood to climate change using life cycle analysis. Global fuel use by fisheries, just to catch and land fish (not process and ship it further), has been estimated as $1.2 \%$ of global oil consumption (Tyedmers et al., 2005). No 
discussion about seafood sustainability should be complete without considering the relationships between its resource dependencies and emissions and the stability of the broader biogeochemical cycles that cumulatively provide the basis for healthy marine ecosystems.

Fish sticks could be made of heavily managed MSCcertified Alaska pollock but the fish might have travelled $6,900 \mathrm{~km}$ before reaching the processing plant. Salmon farmed in Chile, filleted in China, processed and packaged in Canada and eaten by a customer in San Diego could travel as far as $35,800 \mathrm{~km}$ (Grescoe, 2008). In the growing Mediterranean bluefin tuna ranching industry highly threatened fish are taken from the wild and towed back to large cages where they are fattened on herring and other wild-caught forage fish. A full-grown bluefin tuna may include energy costs of finding, catching and towing the tuna, as well as of catching, transporting and freezing the feed fish. The inclusion of life cycle thinking would, at a minimum, underscore the importance of promoting energy-efficient fisheries and aquaculture production, as well as low trophic level culture systems (Pelletier \& Tyedmers, 2008), and industrial fisheries would probably be considered far less sustainable than small-scale fisheries.

\section{Eliminating the wasteful fishmeal industry}

In North America and Europe there has been a call to eat lower on the food web (Pimentel \& Pimentel, 2003; Pollan, 2008) and this has been echoed for marine food webs (Hall, 2007; Grescoe, 2008). However, humans currently must compete with factory-farmed animals for this meal of small pelagic fish. Currently, c. 30 million t of fish (36\% of world fisheries catch) are ground up each year into fishmeal and oil, mostly to feed farmed fish, chicken and pigs (Alder et al., 2008).

We argue that decreasing the amount of fish used for the production of animal feed should be a top priority of the sustainable seafood movement, particularly because pigs and chickens alone consume six and two times the amount of seafood as US and Japanese consumers, respectively. One premise of sustainable seafood should be that no fishery that catches fish for the production of animal or feed fish oil should be eco-certified. Instead, groups interested in promoting sustainable seafood should encourage direct human consumption of forage fish.

In Peru, for instance, the anchovy Engraulis ringens fishery, which began in the early 1950s, now produces half of the world's fishmeal. But, whilst Peru exports fishmeal and other products derived from its catch of 5-10 million $t$ of anchovy each year, half of its population ( 15 million people) lives under conditions of critical poverty and $25 \%$ of infants are malnourished. This discrepancy led to a major campaign in 2006 involving scientists, chefs and politicians to remake the image of the anchovy. The goals were to increase the real and perceived value of these fish, to develop local and export markets and to make investing in freezing and canning facilities attractive so that Peru could afford to shift away from turning perfectly edible fish into fishmeal. During the week dedicated to promoting the anchovy, 18,00o Peruvians tasted the small fish at more than 30 restaurants in Lima, the nation's capital. Anchovies are much more valuable to the Peruvian economy as canned fillets than as fishmeal. One $t$ of fillets is sold for five times the price of $1 \mathrm{t}$ of meal and requires half the fish ( $3 \mathrm{t}$ for $1 \mathrm{t}$ fillets vs $6 \mathrm{t}$ for $1 \mathrm{t}$ meal). The government decided to dedicate $30 \%$ of its annual food security budget, or c. USD 80 million, for programmes that will go to supplying anchovies. As a result, by late 2007, 1 year after the campaign began, demand for fresh anchovies had grown by $46 \%$ and demand for canned anchovies was up by $85 \%$. It is hoped that higher prices for anchovies will limit their use in fishmeal industry, thereby causing an overall reduction in demand for anchovies.

Without fishmeal, protein alternatives for animal feed are needed. There is a new interest in soymeal as well as meals made from mass-producing insects (Ratliff, 2008) as a sustainable protein source to replace fishmeal in fish and livestock feeds, and experimental trials show that a fishmeal alternative, at least partially, may be possible. More importantly, agriculture and aquaculture need to revert back to less intensive systems, requiring only plant-based inputs (Pimentel \& Pimentel, 2003).

\section{Eliminating harmful fisheries subsidies}

Arguably, a powerful market force driving overfishing is fisheries subsidies. Globally, fisheries subsidies are an estimated USD 30-34 billion annually, USD 20 billion of which contributes directly to encouraging excess fishing capacity (e.g. fuel subsidies, boat construction; Sumaila \& Pauly, 2006) and favours industrial fisheries over smallscale, more sustainable ones. Yet the US conservation community's investment in eliminating harmful fisheries subsidies has been $<4 \%$ of the investment into consumeroriented market-based campaigns over the past decade (Jacquet \& Pauly, 2008b). The market-oriented goal of eliminating harmful fisheries subsidies must become a global conservation priority. Efforts by the World Trade Organization to discipline fisheries subsidies should be pursued with greater rigour (Sumaila \& Pauly, 2006).

\section{Setting seafood targets}

Finally, to warrant continued investment, each marketbased conservation programme should be designed so as to produce outcomes and testable results (Sutherland et al., 2004). The sustainable seafood movement must also place more emphasis on measurable results, numeracy, data 
sharing and publishing results. The number of consumers reached by a seafood choice campaign is not a measure of success unless it is accompanied by a measurable improvement in wild fish populations. Simply creating demand for an eco-certified product is not enough unless there is a concurrent decrease in demand for other overfished species. All programmes should be designed with a series of testable outcomes, e.g. a measurable decline in demand for certain species (or overall seafood demand), increases in biomass estimates and overall reduction in harmful subsidies.

Sustainable seafood initiatives must also be integrated and coordinated with others and have a common goal. In the same way that coordinated efforts by the scientific community and governments have determined targets for greenhouse gas emissions, national and international targets are needed for seafood catch and consumption (and at what trophic level) to shift global fisheries to sustainable enterprises. Such an analysis must be regional to take into account national differences in fishing capacity (industrial vs artisanal fleets) and complexity of the markets (local vs global). This analysis could yield an 'optimal sustainable consumption rate' of wild fish and, in turn, determine the rate of increase in aquaculture production and other alternatives to seafood to meet demand.

\section{Discussion}

Seafood is one of the only wild foods (aside from fungi) that westerners eat with any regularity. After more than a decade of market-based sustainable seafood initiatives the demand for this last wild food source is higher than ever and has resulted in further declines of fish populations (Pauly et al., 2002; Worm et al., 2006) and a complementary rapid domestication of marine species (Duarte et al., 2007).

Individuals and groups working to curb overfishing in the 1980 s and 1990 s first attempted to work within regulatory frameworks but became frustrated with the pace of regulatory approaches and the tactics of powerful interest groups. Many abandoned the political process in favour of the market. In 1992 Carl Safina unsuccessfully lobbied for a CITES listing of bluefin tuna, which would have banned the trade of tuna (Grescoe, 2008). Safina later assisted in designing the first seafood wallet card and then formed the Blue Ocean Institute, dedicated to creating a new ethic but also to developing seafood guides and working closely with consumers. These efforts snowballed into the concerted international effort aimed at household consumers today.

We do not argue against the principle that consumers should make a point of choosing products that reflect their ideals, a premise that is especially prevalent in food choices (Clover, 2006; Grescoe, 2008; Pollan, 2008). But, in terms of monetary outlays and human energy, we argue that putting too much emphasis on consumers is not an effective market-based strategy. There is simply too much cheating in the marketplace (e.g. mislabelling), too much misleading information, too many inconsistencies and, so far, too few results.

Just as consumers experienced fatigue in the 1990s after corporate eco-advertising amounted to little action or outcome, so may this decade witness the same fatigue in sustainable seafood campaigns. For instance, in 2002, Sainsbury's in the UK committed to sourcing all its wild fish from sustainable sources by 2010. But after working closely with the MSC, MSC-certified fish only amount to $1 \%$ of total fish sales (Roheim \& Sutinen, 2006). In 2006 WalMart pledged to source all its capture fish from the MSC by 2010 (Gunther, 2006), a goal Wal-Mart is not likely to meet. The 'greenwashing' that corporations were accused of in the 1990 s could turn into a 'bluewashing' today.

However, it is important to note that these corporations are motivated to make these promises because they believe their consumers want them to act. In this way, it is clear that consumers can help drive changes in the market. However, to avoid the bluewashing phenomenon, sustainable seafood campaigns must be goal oriented and communicate whether or not goals are met. National and international seafood consumption targets for specific species and/or overall demand could help determine these goals. To achieve reduction in seafood demand more quickly, sustainable seafood initiatives could work higher in the demand chain, begin connecting seafood to climate change, campaign to divert small fish away from livestock feed and into consumer markets, and work to eliminate harmful fisheries subsidies.

The conservation movement's emphasis on consumers and market-based initiatives is perhaps a result of the Reagan-Thatcher-Bush years, during which government was perceived as the problem and markets as panaceas. Today, this perception is changing. Working with household consumers alone cannot save fish. Although government regulations are far from perfect, the successes of government efforts to improve fisheries have been more obvious and measurable (e.g. marine protected areas). Even within a market-based approach there is a need to ensure that information is correct and that conservation efforts are not obstructed by harmful subsidies. In other words, working with consumers and retailers also brings the imperative of working with governments.

\section{Acknowledgements}

We thank A. Cooper, K. Cramer, M. Powell, K. Rechberger and three anonymous reviewers for their helpful comments, J. Cannon at FishSource who sponsored an earlier version of Table 1 , and the Pew Charitable Trusts for funding The Sea Around Us Project. 


\section{References}

Alder, J., Campbell, B., Karpouzi, V., Kaschner, K. \& PAUly, D. (2008) Forage fish: from ecosystems to markets. Annual Review of Environmental Resources, 33, 7.1-7.14.

Angulo, E. \& Courchamp, F. (2009) Rare species are valued big time. PLoS ONE, 4(4), e5215. Doi:10.1371/journal.pone.0005215

Bridgespan Group (2005) Seafood Choices Evaluation Prepared for the David \& Lucile Packard Foundation. Bridgespan Group, Boston, USA.

Brunner, E.J., Jones, P.J.S., Friel, S. \& Bartley, M. (2009) Fish, human health and marine ecosystem health: policies in collision. International Journal of Epidemiology, 38, 93-100.

Chuenpagdee, R., Liguori, L., Palomares, M.L.D. \& Pauly, D. (2006) Bottom-up, global estimates of small-scale marine fisheries catches. Fisheries Centre Research Reports, 14, 112.

Clover, C. (2006) The End of the Line: How Overfishing is Changing the World and What We Eat. New Press, New York, USA.

Cramer, K.L., Perryman, W.L. \& Gerrodette, T. (2008) Declines in reproductive output in two dolphin populations depleted by the yellowfin tuna purse-seine fishery. Marine Ecological Progress Series, 369, 273-285.

Duarte, C.M., Marba, N. \& Holmer, M. (2007) Rapid domestication of marine species. Science, 316, 382-383.

Environmental Defense Fund (2009) Http://www.edf.org/ page.cfm?tagID $=1521$ [accessed 14 August 2009].

Fiorillo, J. (2008) Loblaw to Expand Eco-Label Fish. Intrafish News Service. Http://www.intrafish.no/global/news/article232637.ece [accessed 7 November 2008].

Froese, R. (2004) Keep it simple: three indicators to deal with overfishing. Fish and Fisheries, 5, 86.

Gault, A., Meinard, Y. \& Courchamp, F. (2008) Less Is More: Rarity Trumps Quality in Luxury Markets. Nature Precedings. Http://precedings.nature.com/documents/169o/version/1 [accessed 2 February 2009].

Gerrodette, T. \& Forcada, J. (2005) Non-recovery of two spotted and spinner dolphin populations in the eastern tropical Pacific Ocean. Marine Ecological Progress Series, 291, 1-21.

Gerrodette, T., Watters, G., Perryman, W. \& Balance, L. (2008) Estimates of 2006 Dolphin Abundance in the Eastern Tropical Pacific with Revised Estimates from 1986-2003. NOAA Technical Memorandum April 2008. NOAA, National Marine Fisheries Service, La Jolla, California.

GREENPEACE (2005) A Recipe for Disaster: Supermarkets' Insatiable Appetite for Seafood. Greenpeace, London, UK. Http:// www.greenpeace.org.uk/files/pdfs/migrated/oceans/supermarkets/ report/supermarkets_seafood.pdf [accessed 12 August 2008].

GREENPEACE (2006) A Recipe for Change: Supermarkets Respond to the Challenge of Sourcing Sustainable Seafood. Greenpeace, London, UK. Http://www.greenpeace.org.uk/media/reports/a-recipe-forchange [accessed 12 August 2008].

Grescoe, T. (2008) Bottomfeeder: How to Eat Ethically in a World of Vanishing Seafood. Bloomsbury, London, UK.

Gulbrandsen, L.H. (2009) The emergence and effectiveness of the Marine Stewardship Council. Marine Policy, 33, 654-660.

Gunther, M. (2006) Saving Seafood. CNNMoney.com, July 31, 2007. Http://money.cnn.com/2006/o7/25/news/companies/pluggedin_ gunther_fish.fortune/index.htm [accessed 12 August 2008].

Hall, M. (2007) Eat more anchovies. Conservation Magazine, 8(3). Http://www.conbio.org/cip/article30713.cfm [accessed 4 April 2008].

Halweil, B. (2006) Catch of the Day: Choosing Seafood for Healthier Oceans. Worldwatch Institute, Washington, DC, USA.

InTERNATIONAL SEAFOOD Guide (2009) Http://www.seafoodguide. org [accessed 14 August 2009].
Jacquet, J. (2007) The Toothfish That Bit Al Gore. The Tyee, Vancouver, Canada. Http://thetyee.ca/Views/2007/07/20/ Toothfish/ [accessed 20 July 2007].

JACQUet, J.L. \& PAUly, D. (2007) The rise of seafood awareness campaigns in an era of collapsing fisheries. Marine Policy, 31, 308-313.

JACQUET, J.L. \& PAULY, D. (2008a) Funding priorities: big barriers to small-scale fisheries. Conservation Biology, 22, 832-835.

Jacquet, J.L. \& Pauly, D. (2008b) Trade secrets: renaming and mislabeling of seafood. Marine Policy, 32, 309-318.

Kaiser, M.J. \& Edwards-Jones, G. (2006) The role of ecolabeling in fisheries management and conservation. Conservation Biology, 20, 393-398.

Kavanagh, P. \& Pitcher, T.J. (2004) Implementing Microsoft Excel software for rapfish: a technique for the rapid appraisal of fisheries status. Fisheries Centre Research Reports, 12.

LeAditter, D. \& WARD, T.J. (2007) An evaluation of systems for the integrated assessment of capture fisheries. Marine Policy, $31,458-469$.

NMFS (National Marine Fisheries Service) (2006) Fisheries of the USA, 2005. NMFS, Silver Springs, USA.

NOAA (National Oceanic and Atmospheric Administration) (2008a) NOAA Finds Decline in Pollock; Recommends Catch Cut to Council. Http://www.noaanews.noaa.gov/stories2008/ 20081120_pollock.html [accessed 20 November 2008].

NOAA (National Oceanic ANd Atmospheric Administration) (2008b) Seafood Consumption Declines Slightly in 2007. Http:// www.noaanews.noaa.gov/stories2008/20080717_seafood.html [accessed 11 November 2008].

Pauly, D., Christensen, V., Guénette, S., Pitcher, T.J., Sumaila, U.R., Walters, C.J. et al. (2002) Towards sustainability in world fisheries. Nature, 418, 689-695.

Pelletier, N. \& Tyedmers, P. (2008) Life cycle considerations for improving sustainability assessments in seafood awareness campaigns. Environmental Management, 42, 918-931.

Pelletier, N., Ayer, N., Tyedmers, P., Kruse, S., Flysjo, A., Robillard, G. et al. (2006) Impact categories for life cycle assessment research of seafood production: review and prospectus. International Journal of Life Cycle Assessment, 12, 414-421.

Pfeiffer, T. \& NowaK, M.A. (2006) All in the game. Nature, 441, 583-584.

Pimentel, D. \& Pimentel, M. (2003) Sustainability of meat-based and plant-based diets and the environment. American Journal of Clinical Nutrition, 78, 660S-663S.

Pitcher, T. (1999) RapFish, A Rapid Appraisal Technique for Fisheries and its Application to the Code of Conduct for Responsible Fisheries. FAO Fisheries Circular, No. 947. Food and Agriculture Organization of the United Nations, Rome, Italy.

Pitcher, T. (2008) The sea ahead: challenges to marine biology from seafood sustainability. Hydrobiologia, 606, 161-185.

Pitcher, T. \& Preikshot, D.B. (2001) RapFish: A rapid appraisal technique to evaluate the sustainability status of fisheries. Fisheries Research, 49, 255-270.

Pollan, M. (2008) In Defense of Food: An Eater's Manifesto. Penguin Press, New York, USA.

Quadra Planning Consultants Ltd (2004) Seafood Watch Evaluation: Summary Report. Galiano Institute for the Environment, Saltspring Island, Canada.

Ratliff, B. (2008) Producers Might Put Fish on Diet of MassProduced Insects. Delta Farm Press. Http://deltafarmpress.com/ mag/farming_producers_put_fish/ [accessed 8 March 2009].

Roheim, C. \& Sutinen, J. (2006) Trade and Marketplace Measures to Promote Sustainable Fishing Practices. International Centre for Trade and Sustainable Development, Paris, France. 
Semmann, D., Krambeck, H.J. \& Milinski, M. (2004) Strategic investment in reputation. Behavioral Ecology and Sociobiology, $56,248-252$.

Sumaila, U.R. \& Pauly, D. (2006) Catching more bait: a bottomup re-estimation of global fisheries subsides. Fisheries Centre Research Reports, 14, 114.

Sutherland, W.J., Pullin, A.S., Dolman, P.M. \& Knight, T.M. (2004) The need for evidence-based conservation. Trends in Ecology \& Evolution, 19, 305-308.

Sutton, D. (2003) An unsatisfactory encounter with the MSC-a conservation perspective. In Eco-labelling in Fisheries: What is it all about? (eds B. Phillips, T. Ward \& C. Chaffee), 114-119. Blackwell Publishing, Oxford, UK.

Tennyson, J.M., Winters, K.S. \& Powell, K. (1997) A fish by any other name: a report on species substitution. Presented at the 22nd Annual Meeting of Seafood Science, Technology Society of the Americas. Biloxi, USA, 6 October 1997. Http://wwt.ifas. ufl.edu/22nd/Ann/fileo8.pdf [accessed 1 May 2007].

Tyedmers, P.H., Watson, R. \& Pauly, D. (2005) Fueling global fishing fleets. Ambio, 34, 635-638.

Wade, P.R., Watters, G.M., Gerrodette, T. \& Reilly, S.B. (2007) Depletion of spotted and spinner dolphins in the eastern tropical Pacific: modeling hypotheses for their lack of recovery. Marine Ecological Progress Series, 343, 1-14.

WARD, T.J. (2008) Barriers to biodiversity conservation in marine fishery certification. Fish and Fisheries, 9, 167-177.

Watson, R. \& Pauly, D. (2001) Systematic distortions in world fisheries catch trends. Nature, 424, 534-536.

WHO (World Health Organization) (2006) Global and Regional Food Consumption Patterns and Trends: Availability and
Consumption of Fish. Http://www.who.int/nutrition/topics/ 3_foodconsumption/en/index $5 . h$ tml [accessed 3 November 2007]. Worm, B., Barbier, E.B., Beaumont, N., Duffy, E., Folke, C., HALPERN, B.S. et al. (2006) Impacts of biodiversity loss on ocean ecosystem services. Science, 314, 787-790.

\section{Biographical sketches}

JENNIFER JACQUET is interested in conservation efforts directed at consumers; she also writes the blog Guilty Planet. JoHn Hocevar is the Oceans Campaign Director for Greenpeace USA, and combines policy, science, non-violent direct action and market approaches for marine protection. SHERM AN L AI is project coordinator for the Lenfest Ocean Futures Project and specializes in building decision support systems that allow non-technical users to understand complex model output. PATRICIA MAJLUF's interests are in developing the enabling conditions for ecosystem-based management of the Humboldt Upwelling ecosystem in Peru and making the anchovy fishery the largest sustainable fishery. Nathan Pelletien's research in ecological economics focuses on the contributions of material and energy flows through global food systems to macro-scale environmental change. Tony Pitcher is a restoration ecologist with research interests in aquatic ecosystems and fisheries; he edits Fish and Fisheries. ENRIC SALA investigates human impacts on ocean ecosystems, focusing mostly on overexploitation. RASHID SUMAILA specializes in bioeconomics and marine ecosystem valuation. D A NIEL PAULY leads the Sea Around Us Project, which studies the impact of global fisheries on marine ecosystems. 